\title{
A Novel Nonlinear Neural Network Ensemble Model for Financial Time Series Forecasting
}

\author{
Kin Keung Lai ${ }^{1,2}$, Lean $\mathrm{Yu}^{2,3}$, Shouyang Wang ${ }^{1,3}$, and Huang $\mathrm{Wei}^{4}$ \\ ${ }^{1}$ College of Business Administration, Hunan University, Changsha 410082, China \\ ${ }^{2}$ Department of Management Sciences, City University of Hong Kong, \\ Tat Chee Avenue, Kowloon, Hong Kong \\ \{mskklai, msyulean\} @cityu.edu.hk \\ ${ }^{3}$ Institute of Systems Science, Academy of Mathematics and Systems Science, \\ Chinese Academy of Sciences, Beijing 100080, China \\ \{yulean, sywang\} @amss.ac.cn \\ ${ }^{4}$ School of Management, Huazhong University of Science and Technology, \\ 1037 Luoyu Road, Wuhan 430074, China
}

\begin{abstract}
In this study, a new nonlinear neural network ensemble model is proposed for financial time series forecasting. In this model, many different neural network models are first generated. Then the principal component analysis technique is used to select the appropriate ensemble members. Finally, the support vector machine regression method is used for neural network ensemble. For further illustration, two real financial time series are used for testing.
\end{abstract}

\section{Introduction}

Financial market is a complex evolved dynamic market with high volatility and noise. Due to its irregularity, financial time series forecasting is regarded as a rather challenging task. For traditional statistical methods, it is extremely difficult to capture the irregularity. In the past decades, many emerging techniques, such as neural networks, were widely used in the financial time series forecasting and obtained good results.

However, neural networks are a kind of unstable learning methods, i.e., small changes in the training set and/or parameter selection can produce large changes in the predicted output. This diversity of neural networks is a naturally by-product of the randomness of the inherent data and training process, and also of the intrinsic nonidentifiability of the model. For example, the results of many experiments have shown that the generalization of single neural network is not unique. That is, the neural network's results are not stable. Even for some simple problems, different structures of neural networks (e.g., different number of hidden layers, different hidden nodes and different initial conditions) result in different patterns of network generalization. In addition, even the most powerful neural network model still cannot cope well when dealing with complex data sets containing some random errors or insufficient training data. Thus, the performance for these data sets may not be as good as expected [1-2].

Recently, some experiments have been proved that neural network ensemble forecasting model is an effective approach to the development of a high performance forecasting system relative to single neural networks [3]. Meantime, some linear 
ensemble methods are also presented [4-6]. Different from the previous work, this study proposes a novel nonlinear ensemble forecasting method in terms of support vector machine regression principle.

The rest of this study is organized as follows. Section 2 describes the building process of the nonlinear neural network ensemble forecasting model in detail. For further illustration, two real financial time series are used for testing in Section 3. Finally, some concluding remarks are drawn in Section 4.

\section{The Building Process of the Nonlinear Ensemble Model}

In this section, a triple-phase nonlinear neural network ensemble model is proposed for financial time series forecasting. First of all, many individual neural predictors are generated. Then an appropriate number of neural predictors are selected from the considerable number of candidate predictors. Finally, selected neural predictors are combined into an aggregated neural predictor in a nonlinear way.

\section{A. Generating individual neural network predictors}

With the work about bias-variance trade-off of Breiman [7], an ensemble model consisting of diverse models with much disagreement is more likely to have a good generalization. Therefore, how to generate diverse models is a crucial factor. For neural network model, there are four methods for generating diverse models.

(1) Initializing different starting weights for each neural network models.

(2) Training neural networks with different training subsets.

(3) Varying the architecture of neural network, e.g., changing the different numbers of layers or different numbers of nodes in each layer.

(4) Using different training algorithms, such as the back-propagation algorithm, radial basis function algorithm and Bayesian regression algorithms.

\section{B. Selecting appropriate ensemble members}

After training, each individual neural predictor has generated its own result. However, if there are a great number of individual members, we need to select a subset of representatives in order to improve ensemble efficiency. In this study, the principal component analysis (PCA) technique [8] is adopted to select appropriate ensemble members. Interested readers can be referred to [8] for more details.

\section{Combining the selected members}

Depended upon the work done in previous phases, a collection of appropriate ensemble members can be collected. The subsequent task is to combine these selected members into an aggregated predictor in an appropriate ensemble strategy. Generally, there are two ensemble strategies: linear ensemble and nonlinear ensemble.

Typically, linear ensemble strategy includes two approaches: the simple averaging [4] approach and the weighted averaging [5] approach. There are two types of weighted averaging: the mean squared error (MSE) based regression approach [6] and variance-based weighted approach [6]. The nonlinear ensemble strategy is a promising approach for determining the optimal neural ensemble predictor's weight. The literature only mentions one nonlinear approach: neural network-based nonlinear 
ensemble method [8]. Different from the previous work, we propose a new nonlinear ensemble method with support vector machine regression (SVMR) [9] principle.

Generally speaking, an SVMR-based nonlinear ensemble forecasting model can be viewed as a nonlinear information processing system that can be represented as:

$$
\hat{y}=f\left(\hat{x}_{1}, \hat{x}_{2}, \cdots, \hat{x}_{n}\right)
$$

where $\left(\hat{x}_{1}, \hat{x}_{2}, \cdots, \hat{x}_{n}\right)$ is the output of individual neural network predictors, $\hat{y}$ is the aggregated output, $f(\cdot)$ is nonlinear function determined by SVMR. In this sense, SVMR-based ensemble is a nonlinear ensemble method.

\section{Empirical Analysis}

The data set used for our experiment consists of two time series data: the S\&P 500 index series, and the GBP/USD series. The data used in this study are obtained from Datastream (http://www.datastream. com). The entire data set covers the period from January 1, 1991 to December 31, 2002. We take daily data from January 1, 1991 to December 31, 2000 as the in-sample data sets and take the data from January 1, 2001 to December 31, 2002 as the out-of-sample data set (i.e., testing set), which are used to evaluate the good or bad performance of predictions. The root mean squared error $(R M S E)$ is used the evaluation criteria over each of the two different testing sets and corresponding results are reported in Tables 1 .

Table 1. A comparison of RMSE between different ensemble methods

\begin{tabular}{ccccc}
\hline \multirow{2}{*}{ Ensemble Method } & \multicolumn{2}{c}{ S\&P500 } & \multicolumn{2}{c}{ GBP/USD } \\
\cline { 2 - 5 } & RMSE & Rank & RMSE & Rank \\
\hline Simple averaging & 0.0115 & 3 & 0.0075 & 4 \\
MSE regression & 0.0159 & 5 & 0.0078 & 5 \\
Variance-based weight & 0.0124 & 4 & 0.0058 & 3 \\
Neural network & 0.0108 & 2 & 0.0044 & 2 \\
SVMR & 0.0098 & 1 & 0.0017 & 1 \\
\hline
\end{tabular}

From Table 1, we can conclude that (1) in all the ensemble methods the SVMRbased ensemble model performs the best, followed by the neural network based ensemble method and other three linear ensemble method from a general view and (2) the nonlinear ensemble methods including neural network-based and SVMR-based method outperform all the linear ensemble methods, indicating that the nonlinear ensemble methods are more suitable for financial time series forecasting than the linear ensemble approaches due to high volatility of the financial time series. Interestedly, in the testing case of S\&P 500, the simple averaging ensemble method can beat other two linear ensemble approaches. The phenomenon also reflects a basic principle, i.e., the simplest may be the best. 


\section{Conclusions}

In this study, we propose a novel triple-phase nonlinear ensemble predictor for financial time series forecasting. The experimental results reported in this paper demonstrate the effectiveness of the proposed nonlinear ensemble approach, implying that the proposed nonlinear ensemble model can be used as a feasible approach to financial time series forecasting.

\section{Acknowledgements}

This work is partially supported by National Natural Science Foundation of China (NSFC No. 70221001); Chinese Academy of Sciences; Key Research Institute of Humanities and Social Sciences in Hubei Province-Research Center of Modern Information Management and Strategic Research Grant of City University of Hong Kong (SRG No. 7001677).

\section{References}

1. Naftaly, U, Intrator, N, Horn, D.: Optimal Ensemble Averaging of Neural Networks. Network Computation in Neural Systems 8 (1997) 283-296

2. Carney, J, Cunningham, P.: Tuning Diversity in Bagged Ensembles. International Journal of Neural Systems 10 (2000) 267-280

3. Bishop, C.M.: Neural Networks for Pattern Recognition. Oxford University Press (1995)

4. Benediktsson, J.A., Sveinsson, J.R., Ersoy, O.K., Swain, P.H.: Parallel Consensual Neural Networks. IEEE Transactions on Neural Networks 8 (1997) 54-64

5. Perrone, M.P., Cooper, L.N.: When Networks Disagree: Ensemble Methods for Hybrid Neural Networks. In Mammone, R.J. (ed.): Neural Networks for Speech and Image Processing, Chapman-Hall (1993) 126-142

6. Krogh, A., Vedelsby, J.: Neural Network Ensembles, Cross Validation, and Active Learning. In: Tesauro, G., Touretzky, D., Leen, D. (eds.): Advances in Neural Information Processing Systems. The MIT Press (1995) 231-238

7. Breiman, L. Combining Predictors. In: Sharkey, A.J.C. (ed.): Combining Artificial Neural Nets - Ensemble and Modular Multi-net Systems. Springer, Berlin (1999) 31-50

8. Yu, L., Wang, S.Y., Lai, K.K.: A Novel Nonlinear Ensemble Forecasting Model Incorporating GLAR and ANN for Foreign Exchange Rates. Computers and Operations Research 32 (2005) 2523-2541

9. Vapnik, V.: The Nature of Statistical Learning Theory. New York: Springer-Verlag (1995) 